\title{
On the Solutions of Fractional Cauchy Problem Featuring Conformable Derivative
}

\author{
Mehmet Yavuz ${ }^{1, *}$, and Necati Özdemir ${ }^{2}$ \\ ${ }^{1}$ Department of Mathematics-Computer Sciences, Necmettin Erbakan University, Konya, Turkey \\ ${ }^{2}$ Department of Mathematics, Balikesir University, Balikesir, Turkey
}

\begin{abstract}
In this study, we have obtained analytical solutions of fractional Cauchy problem by using q-Homotopy Analysis Method (q-HAM) featuring conformable derivative. We have considered different situations according to the homogeneity and linearity of the fractional Cauchy differential equation. A detailed analysis of the results obtained in the study has been reported. According to the results, we have found out that our obtained solutions approach very speedily to the exact solutions.
\end{abstract}

\section{Introduction}

In recent years, mathematical modelling with fractional PDEs in some special areas such like physics, mathematics, engineering, finance, biology and medicine [1-8] have been studied. Analytical solutions for fractional differential equations (FDEs) are not very popular in the literature, but the methods applied to avoid effective engineering analyzes, and therefore, numerical methods are frequently applied [9-13].

Recently, [14-16] recommended a new derivative operator namely "conformable" (CDO) and some applications of this operator in various fields have been improved. In this context, some researchers [5, 7, 17-20] applied the conformable operator to solve the problems in engineering, finance, biology, medicine, physics and applied mathematics. In this study, we have solved the linear/nonlinear fractional Cauchy problem with the proposed q-HAM described by using the conformable operator.

\section{Fundamental Properties of the Conformable Operator}

Definition 1. Let $u:[0, \infty) \rightarrow \mathbb{R}$ be a function. The conformable derivative of $u$ order $\alpha \in(0,1]$ is defined by [14]:

$$
C D_{t}^{\alpha}(u)(t)=\lim _{\varepsilon \rightarrow 0} \frac{u\left(t+\varepsilon t^{1-\alpha}\right)-u(t)}{\varepsilon}
$$

for all $t>0$.

\footnotetext{
*Corresponding author: mehmetyavuz@konya.edu.tr
} 
Definition 2. $[14,16]$ The $\alpha$-fractional integral of $u$ function starting is defined by

$$
I_{a}^{\alpha}(u)(t)=I_{a}^{1}\left(t^{\alpha-1} u\right)=\int_{a}^{t} \frac{u(x)}{x^{1-\alpha}} d x, \quad \alpha \in(0,1) .
$$

\section{3 q-Homotopy Analysis Method in the Conformable Sense}

Let us take the nonlinear FPDE:

$$
\mathcal{\alpha}\left(C D_{t}^{\alpha} u(x, t)\right)=f(x, t) \text {, }
$$

where $C D_{t}^{\alpha}$ shows the conformable derivative of order $\alpha$. We show the nonlinear term with $\mathcal{\gamma}$, the known function with $f(x, t)$, and the unknown function with $u(x, t)$. We construct the zero-order modified equation which is related with standard homotopy method as [21]:

$$
(1-n q) L\left(\omega(x, t ; q)-u_{0}(x, t)\right)=q h H(x, t)\left(\mathcal{\alpha r}\left(C D_{t}^{\alpha} \omega(x, t ; q)\right)-f(x, t)\right),
$$

where $n \geq 1, q \in[0,1 / n]$ represents the embedded parameter, $L$ is a linear operator, $H(x, t)$ is non-zero supportive function. Distinctly, since $q=0$ and $q=1 / n$, we can see Eq.(4) as

$$
\omega(x, t ; 0)=u_{0}(x, t), \quad \omega\left(x, t ; \frac{1}{n}\right)=u(x, t),
$$

respectively. Therefore, $q$ increases from zero to $1 / n$, the solution $\omega(x, t ; q)$ varies from the initial value $u_{0}(x, t)$ to the solution $u(x, t)$. If the parameters are chosen appropriately, solution of Eq.(5) is available. Now, we have the following expansion of $\omega(x, t ; q)$

$$
\omega(x, t ; q)=u_{0}(x, t)+\sum_{m=1}^{\infty} u_{m}(x, t) q^{m},
$$

where

$$
u_{m}(x, t)=\left.\frac{1}{m !} \frac{\partial^{m} \omega(x, t ; q)}{\partial q^{m}}\right|_{q=0}
$$

After that we get

$$
u(x, t)=u_{0}(x, t)+\sum_{m=1}^{\infty} u_{m}(x, t)\left(\frac{1}{n}\right)^{m}
$$

Let the vector

$$
\vec{u}_{n}=\left\{u_{0}(x, t), u_{1}(x, t), \ldots, u_{n}(x, t)\right\} .
$$

Then we have $m$ th- order altered equation as [22]

$$
L\left[u_{m}(x, t)-\chi_{m}^{*} u_{m-1}(x, t)\right]=h H(x, t) \Re_{m}\left(\vec{u}_{m-1}(x, t)\right)
$$

with initial conditions

$$
u_{m}^{(k)}(x, 0)=0, \quad k=0,1,2, \ldots, m-1,
$$

where

$$
\Re_{m}\left(\vec{u}_{m-1}(x, t)\right)=\left.\frac{1}{(m-1) !} \frac{\partial^{m-1}\left[\mathcal{\gamma}\left(C D_{t}^{\alpha} \omega(x, t ; q)\right)-f(x, t)\right]}{\partial q^{m-1}}\right|_{q=0}
$$

and 


$$
\chi_{m}^{*}= \begin{cases}0, & m \leq 1 \\ n, & m>1 .\end{cases}
$$

Finally, we get the components of solution series as,

$$
u_{m}(x, t)=\chi_{m}^{*} u_{m-1}(x, t)-\chi_{m}^{*} u_{m-1}\left(x, 0^{+}\right)+h H(x, t) \mathfrak{I}_{t}^{\alpha} \mathfrak{R}_{m}\left(\vec{u}_{m-1}(x, t)\right) .
$$

\section{Numerical Examples}

Example 1. We consider a special case of fractional Cauchy equation [23, 24]

$$
C D_{t}^{\alpha} u(x, t)+u_{x}(x, t)=x, \quad x \in R, \quad t>0, \quad 0<\alpha \leq 1,
$$

with initial condition

$$
u(x, 0)=e^{x}, \quad x \in R .
$$

If we choose $H(x, t)=1$, we can construct the zeroth-order deformation equation as

$$
L\left[u_{m}(x, t)-\chi_{m}^{*} u_{m-1}(x, t)\right]=h \Re_{m}\left(\vec{u}_{m-1}(x, t)\right),
$$

with initial condition for $m \geq 1, u_{m}(x, 0)=0, \chi_{m}^{*}$ is as defined in (13) and

$$
\Re_{m}\left(\vec{u}_{m-1}(x, t)\right)=C D_{t}^{\alpha} u_{m-1}+\left(u_{m-1}\right)_{x}-x .
$$

Therefore, the solution of Eq.(15) for $m \geq 1$ becomes

$$
u_{m}(x, t)=\chi_{m}^{*} u_{m-1}(x, t)+h \mathfrak{I}_{t}^{\alpha} \Re_{m}\left(\vec{u}_{m-1}(x, t)\right) .
$$

Then using Eq.(19) we can obtain the q-HAM consequence as

$$
\begin{aligned}
u_{0}(x, t)= & e^{x}, \\
u_{1}(x, t)= & h\left(e^{x}-x\right) \frac{t^{\alpha}}{\alpha} \\
u_{2}(x, t)= & n h\left(e^{x}-x\right) \frac{t^{\alpha}}{\alpha}+h^{2}\left(e^{x}-x\right) \frac{t^{\alpha}}{\alpha}-h x \frac{t^{\alpha}}{\alpha}+h^{2}\left(e^{x}-1\right) \frac{t^{2 \alpha}}{2 \alpha^{2}}, \\
u_{3}(x, t)= & \left(n^{2} h+2 n h^{2}+h^{3}\right)\left(e^{x}-x\right) \frac{t^{\alpha}}{\alpha}-\left(h^{2}+n h+h\right) x \frac{t^{\alpha}}{\alpha} \\
& +\left(2 n h^{2}+2 h^{3}\right)\left(e^{x}-1\right) \frac{t^{2 \alpha}}{2 \alpha^{2}}-h^{2} \frac{t^{2 \alpha}}{2 \alpha^{2}}+h^{3} e^{x} \frac{t^{3 \alpha}}{6 \alpha^{3}},
\end{aligned}
$$

The series solution of Eq.(15) by q-HAM can be considered as

$$
u(x, t ; n ; h)=e^{x}+\sum_{m=1}^{\infty} u_{m}(x, t ; n ; h)\left(\frac{1}{n}\right)^{m} .
$$

For special values of $h=-1, n=1$ and $\alpha=1$, we get the exact solution of Eq.(15) as

$$
u(x, t)=e^{x-t}+x t-\frac{t^{2}}{2} \text {. }
$$

Example 2. Consider the following fractional nonlinear inviscid Burgers' equation [23, 24]

$$
C D_{t}^{\alpha} u(x, t)+u(x, t) u_{x}(x, t)=0, \quad x \in R, \quad t>0, \quad 0<\alpha \leq 1,
$$

subject to the initial condition

$$
u(x, 0)=x, \quad x \in R .
$$

We can use the nonlinear operator for our problem as

$$
\mathcal{\gamma}[\omega(x, t ; q)]=C D_{t}^{\alpha} \omega(x, t ; q)+\omega(x, t ; q) \omega_{x}(x, t ; q) \text {. }
$$


Following similar steps with Example 1, we construct the q-HAM iterations as below

$$
\begin{aligned}
& u_{0}(x, t)=x, \\
& u_{1}(x, t)=h x \frac{t^{\alpha}}{\alpha}, \\
& u_{2}(x, t)=n h x \frac{t^{\alpha}}{\alpha}+h^{2} x \frac{t^{\alpha}}{\alpha}+2 h^{2} x \frac{t^{2 \alpha}}{2 \alpha^{2}}, \\
& u_{3}(x, t)=\left(n^{2} h+2 n h^{2}+h^{3}\right) x \frac{t^{\alpha}}{\alpha}+\left(4 n h^{2}+4 h^{3}\right) x \frac{t^{2 \alpha}}{2 \alpha^{2}}+h^{3} x \frac{t^{3 \alpha}}{\alpha^{3}},
\end{aligned}
$$

An appropriate solution of Eq.(23) in terms of $h$ and $n$ is given

$$
u(x, t ; n ; h)=x+\sum_{m=1}^{\infty} u_{m}(x, t ; n ; h)\left(\frac{1}{n}\right)^{m} .
$$

For special values of $h=-1, n=1$ and $\alpha=1$, we have the exact solution of Eq.(23) as

$$
u(x, t)=\frac{x}{1-t} .
$$

In Figures below, the plots of solution functions of Eq.(23) for different values of $\alpha, h, n, t$ and $x$ are presented.
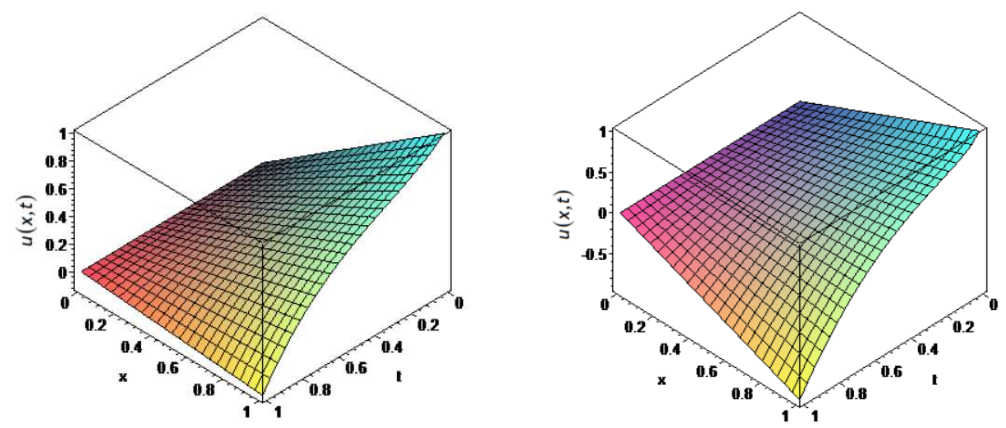

Fig.1. q-HAM Solutions of Eq.(23) with $\alpha=0.75$ (left) $\alpha=0.99$ (right)
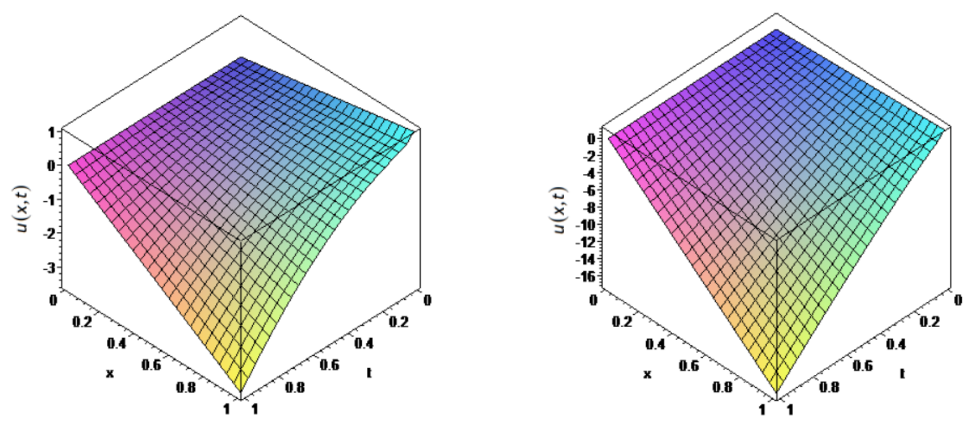

Fig.2. q-HAM Solutions of Eq.(23) with $\alpha=0.55$ (left) $\alpha=0.35$ (right) 

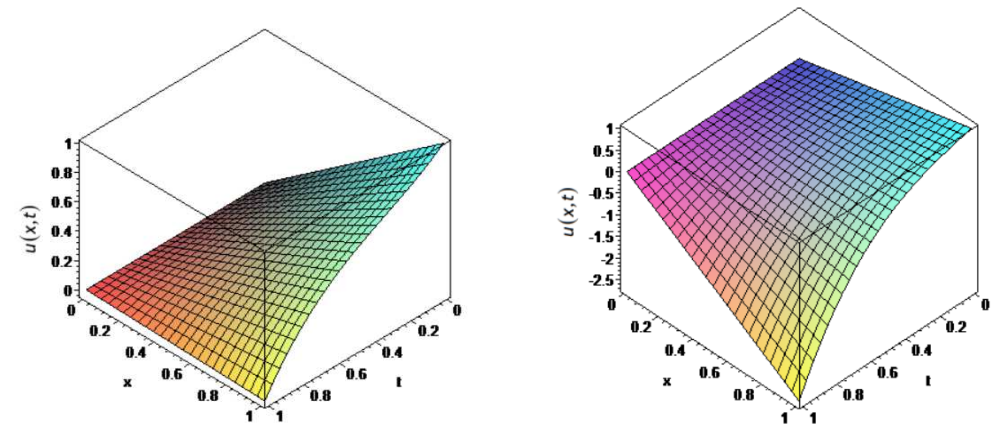

Fig.3. q-HAM Solutions of Eq.(23) for $n=0.99$ (left) $n=0.7$ (right)
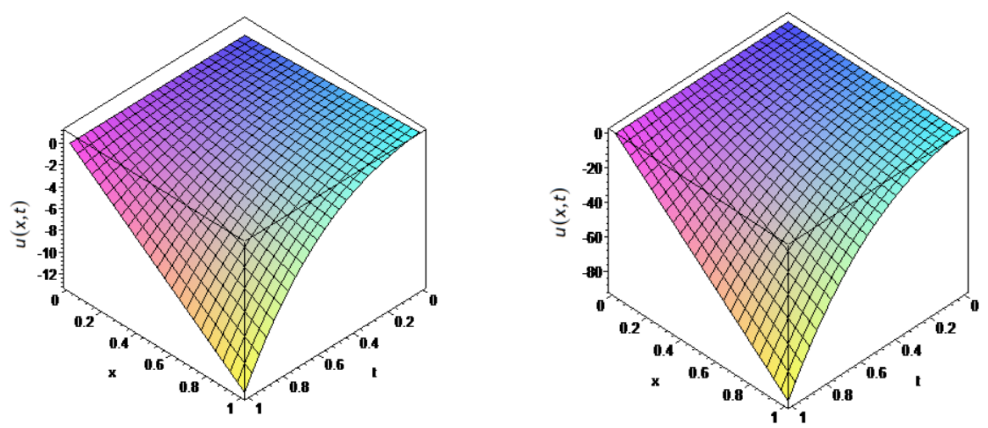

Fig.4. q-HAM Solutions of Eq.(23) for $n=0.5$ (left) $n=0.3$ (right)
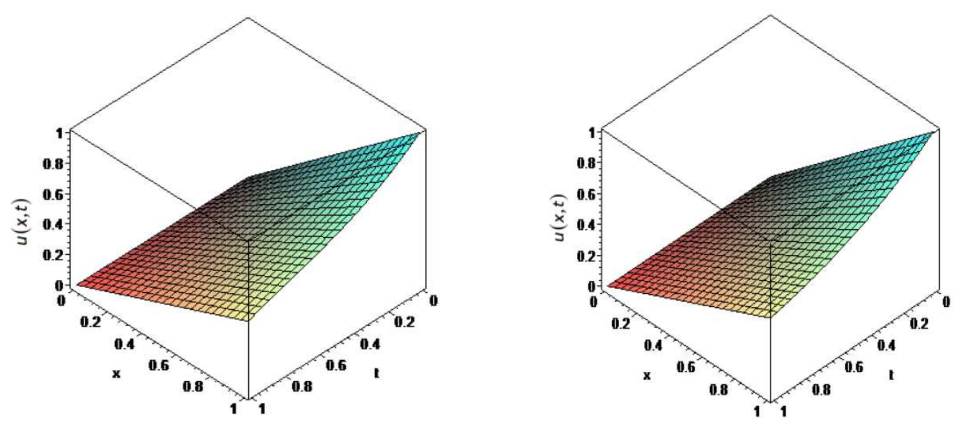

Fig.5. q-HAM Solutions of Eq.(23) for $h=-0.3$ (left) $h=-0.5$ (right)
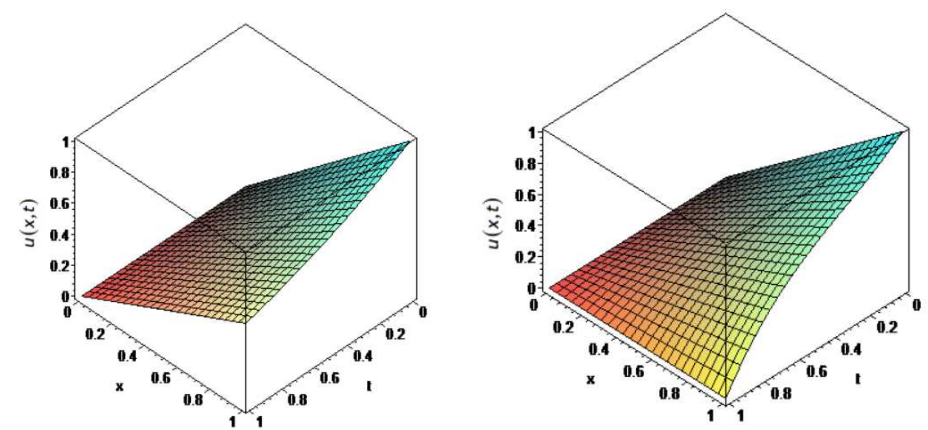

Fig.6. q-HAM Solutions of Eq.(23) for $h=-0.7$ (left) $h=-1$ (right) 


\section{Conclusions}

In this study, we have considered the solutions of linear/nonlinear fractional PDEs by using the q-HAM that is a generalized form of HAM. We have redefined the suggested method with CDO to get the solutions of the fractional Cauchy problems and have applied it to the mentioned problems easily. Then we have verified the efficiencies and correctness of the recommended method by drawing the figures for different values of $\alpha, n, h, x$ and $t$. Moreover, the results show that the q-HAM solutions when $n=1$, give HAM solutions and when the auxiliary parameter is equal to -1 , give the classical HPM solutions. The successful results of the mentioned method show that the method is in complete coherent with the exact solutions even for considering a nonlinear fractional partial differential equation.

\section{References}

1. R. Hilfer, Applications of fractional calculus in physics. Applications of Fractional Calculus in Physics. Edited by HILFER R. Published by World Scientific Publishing Co. Pte. Ltd.,. ISBN\# 9789812817747, (2000).

2. E. Bonyah, A. Atangana, M.A. Khan, Modeling the spread of computer virus via Caputo fractional derivative and the beta-derivative. Asia Pacific Journal on Computational Engineering, 4(1), p. 1. (2017).

3. H. Bulut, H.M. Baskonus, F.B.M. Belgacem, The analytical solution of some fractional ordinary differential equations by the Sumudu transform method. Abstract and Applied Analysis, (2013).

4. H.M. Baskonus, T. Mekkaoui, Z. Hammouch, H. Bulut, Active control of a chaotic fractional order economic system. Entropy, 17(8), pp. 5771-5783, (2015).

5. M. Yavuz, N. Özdemir, New Numerical Techniques for Solving Fractional Partial Differential Equations in Conformable Sense. In: Ostalczyk P., Sankowski D., Nowakowski J. (eds) Non-Integer Order Calculus and its Applications. Lecture Notes in Electrical Engineering, vol 496. (2019), Springer, Cham

6. M. Inc, M.T. Gencoglu, A. Akgül, Application of Extended Adomian Decomposition Method and Extended Variational Iteration Method to Hirota-Satsuma Coupled KdV Equation. Journal of Advanced Physics, 6(2), pp. 216-222, (2017).

7. M. Yavuz, N. Ozdemir, H.M. Baskonus, Solutions of partial differential equations using the fractional operator involving Mittag-Leffler kernel. The European Physical Journal Plus, 133(6), pp. 215, (2018).

8. M.G. Sakar, F. Erdogan, The homotopy analysis method for solving the time-fractional Fornberg-Whitham equation and comparison with Adomian's decomposition method. Applied Mathematical Modelling, 37(20-21), pp. 8876-8885, (2013).

9. M. Yavuz, N. Ozdemir, Numerical inverse Laplace homotopy technique for fractional heat equations. Thermal Science, 22(1), pp. 185-194, (2018).

10. N. Özdemir, M. Yavuz, Numerical Solution of Fractional Black-Scholes Equation by Using the Multivariate Padé Approximation. Acta Physica Polonica A, 132(3), 1050-1053, (2017).

11. J. Hristov, Approximate solutions to fractional subdiffusion equations. The European Physical Journal Special Topics, 193(1), pp. 229-243, (2011).

12. A. Yokuş, Numerical Solutions of Time Fractional Korteweg--de Vries Equation and Its Stability Analysis. Communications Faculty of Sciences University of Ankara Series A1 Mathematics and Statistics. 68(1), pp. 353-361, (2019).

13. A. Yokus, D. Kaya, Numerical and exact solutions for time fractional Burgers' equation. Journal of Nonlinear Sciences and Applications, 10(7), pp. 3419-3428, (2017).

14. R. Khalil, M. Al Horani, A. Yousef, M. Sababhehet, A new definition of fractional derivative. Journal of Computational and Applied Mathematics, 264, p. 65-70, (2014). 
15. A. Atangana, D. Baleanu, A. Alsaedi, New properties of conformable derivative. Open Mathematics, 13(1), pp. 889-898, (2015).

16. T. Abdeljawad, On conformable fractional calculus. Journal of computational and Applied Mathematics, 279, pp. 57-66, (2015).

17. D. Avci, B.B. Iskender Eroglu, N. Ozdemir, Conformable Heat Equation on a Radial Symmetric Plate. Thermal Science, 21(2), pp. 819-826, (2017).

18. F. Evirgen, Conformable fractional gradient based dynamic system for constrained optimization problem. Acta Physica Polonica A, 132(3), pp. 1066-1069, (2017).

19. M. Yavuz, N. Özdemir, A different approach to the European option pricing model with new fractional operator. Mathematical Modelling of Natural Phenomena, 13(1), pp. 1-12, (2018).

20. M. Yavuz, Novel solution methods for initial boundary value problems of fractional order with conformable differentiation. An International Journal of Optimization and Control, 8(1), pp. 1-7, (2018).

21. O.S. Iyiola, G.O. Ojo, On the analytical solution of Fornberg-Whitham equation with the new fractional derivative. Pramana, 85(4), pp. 567-575, (2015).

22. S.-J. Liao, An approximate solution technique not depending on small parameters:A special example. International Journal of Non-Linear Mechanics, 30(3), pp. 371-380, (1995).

23. A. Tveito, R. Winther, Introduction to partial differential equations: a computational approach. Vol. 29, (Springer Science \& Business Media 2004).

24. N.H. Asmar, Partial differential equations with Fourier series and boundary value problems. (Courier Dover Publications 2016). 